\title{
Proteomic Discovery of Biomarkers to Predict Prognosis of High-Grade Serous Ovarian Carcinoma
}

\author{
Se Ik Kim ${ }^{1,+} \oplus$, Minsun Jung ${ }^{2,+} \mathbb{C}$, Kisoon Dan ${ }^{3}$, Sungyoung Lee ${ }^{4}$, Cheol Lee ${ }^{2}$,

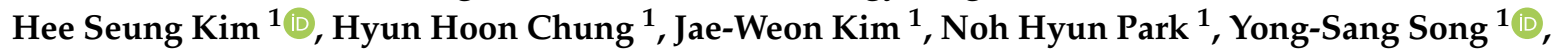 \\ Dohyun Han ${ }^{3, * \mathbb{D}}$ and Maria Lee ${ }^{1, * \mathbb{D}}$ \\ 1 Department of Obstetrics and Gynecology, Seoul National University College of Medicine, Seoul 03080, \\ Korea; seikky@naver.com (S.I.K.); bboddi0311@gmail.com (H.S.K.); chhkmj@gmail.com (H.H.C.); \\ kjwksh@snu.ac.kr (J.-W.K.); pnhkhr@snu.ac.kr (N.H.P.); yssong@snu.ac.kr (Y.-S.S.) \\ 2 Department of Pathology, Seoul National University College of Medicine, Seoul 03080, Korea; \\ jjunglammy@gmail.com (M.J.); fejhh@hanmail.net (C.L.) \\ 3 Proteomics Core Facility, Biomedical Research Institute, Seoul National University Hospital, Seoul 03082, \\ Korea; kisoona@snuh.org \\ 4 Center for Precision Medicine, Seoul National University Hospital, Seoul 03080, Korea; biznok@snu.ac.kr \\ * Correspondence: hdh03@snu.ac.kr (D.H.); marialeemd@gmail.com (M.L.); Tel.: +82-2-2072-1719 (D.H.); \\ $+82-2-2072-2842$ (M.L.) \\ + These two authors contributed equally as first authors.
}

Received: 30 January 2020; Accepted: 24 March 2020; Published: 26 March 2020

check for

\begin{abstract}
Initial identification of biomarkers predicting the exact prognosis of high-grade serous ovarian carcinoma (HGSOC) is important in precision cancer medicine. This study aimed to investigate prognostic biomarkers of HGSOC through proteomic analysis. We conducted label-free liquid chromatography-mass spectrometry using chemotherapy-naïve, fresh-frozen primary HGSOC specimens, and compared the results between a favorable prognosis group (progression-free survival (PFS) $\geq 18$ months, $n=6$ ) and a poor prognosis group (PFS $<18$ months, $n=6$ ). Among 658 differentially expressed proteins, 288 proteins were upregulated in the favorable prognosis group and 370 proteins were upregulated in the poor prognosis group. Using hierarchical clustering, we selected $\alpha 1$-antitrypsin (AAT), nuclear factor-kB (NFKB), phosphomevalonate kinase (PMVK), vascular adhesion protein 1 (VAP1), fatty acid-binding protein 4 (FABP4), platelet factor 4 (PF4), apolipoprotein A1 (APOA1), and $\alpha 1$-acid glycoprotein (AGP) for further validation via immunohistochemical (IHC) staining in an independent set of chemotherapy-naïve primary HGSOC samples $(n=107)$. Survival analyses revealed that high expression of AAT, NFKB, and PMVK were independent biomarkers for favorable PFS. Conversely, high expression of VAP1, FABP4, and PF4 were identified as independent biomarkers for poor PFS. Furthermore, we constructed models predicting the 18-month PFS by combining clinical variables and IHC results. Through leave-one-out cross-validation, the optimal model was based on initial serum CA-125, germline BRCA1/2 mutations, residual tumors after surgery, International Federation of Gynecology and Obstetrics (FIGO) stage, and expression levels of the six proteins. The present results elucidate the proteomic landscape of HGSOC and six protein biomarkers to predict the prognosis of HGSOC.
\end{abstract}

Keywords: ovarian neoplasms; high-grade serous carcinoma; proteomics; immunohistochemistry; prognosis

\section{Introduction}

Ovarian cancer, one of the most fatal gynecologic malignancies, is a global burden with 295,414 new cases and 184,799 deaths estimated each year [1]. Ovarian cancer is the fifth leading cause of 
female cancer-related deaths in the United States [2]. The predominant histologic type is high-grade serous ovarian carcinoma (HGSOC) [3], for which aggressive cytoreductive surgery followed by taxane- and platinum-based chemotherapy is an established standard of care $[4,5]$. Although the initial response rate is high, patients with HGSOC, especially those at advanced stages, eventually experience relapse [6]. In the era of precision medicine, it is important to initially identify biomarkers to accurately predict the exact prognosis of HGSOC to facilitate personalized treatment.

Mass spectrometry (MS)-based proteomics has been widely used to characterize molecular components and underlying mechanisms associated with various malignancies such as colorectal [7], breast [8], lung [9], and ovarian cancers [10-12]. Currently, this emerging technology is used for high-throughput analysis for simultaneous quantification of numerous proteins and discovery of prognostic biomarkers in individual samples. The Clinical Proteomic Tumor Analysis Consortium (CPTAC) provides proteogenomic insights into HGSOC by performing extensive proteomic profiling and correlating results with data contained in The Cancer Genome Atlas (TCGA) database [10]. However, these biomarkers need to be subjected to specific validation studies before being clinically applied.

In this study, we performed label-free quantitative proteomic analysis of chemotherapy-naïve, fresh-frozen primary ovarian cancer tissues to elucidate prognostic protein biomarkers of HGSOC. We then validated our findings via immunohistochemical (IHC) staining in an independent dataset.

\section{Results}

\subsection{Patient Characteristics in the Proteomic Analysis}

Clinicopathologic characteristics of 12 patients with HGSOC, for whom proteomic analysis was performed, are presented in Table S1. Mean patient age was 56.5 years (range 42.0-74.1 years). No differences in patient age, menopausal status, or family history of breast cancer were observed between the good and poor prognosis groups. Initial serum levels of CA-125, International Federation of Gynecology and Obstetrics (FIGO) stage, and residual tumor after primary debulking surgery (PDS) were also similar between the two groups. Of the 12 patients, 4 and 2 patients harbored germline $B R C A 1$ and $B R C A 2$ mutations, respectively, while the other 6 patients harbored wild-type $B R C A 1 / 2$. The median length of observations was 58.1 months, during which 11 patients $(91.7 \%)$ experienced disease recurrence. Patients in the good prognosis group had significantly better progression-free survival (PFS) than those in the poor prognosis group (median, 26.0 vs. 16.9 months; $p=0.001$ ) (Figure S1).

\subsection{Results of Proteomic and Bioinformatic Analyses}

\subsubsection{Global Proteomic Analysis of Ovarian Cancer Tissues}

To identity prognostic biomarkers for HGSOC, we performed MS-based label-free quantification using chemotherapy-naïve, fresh-frozen cancer tissues resected from the primary (non-metastatic) ovarian mass intraoperatively during the debulking surgery $(n=12)$; the good and poor prognosis groups contained six patients per group (Figure 1A). To expand the coverage of the identified ovarian proteome, we used pooled samples to generate a spectral library including 8520 protein groups corresponding to 93,355 unique peptides (Table S2). In the individual samples, 7839 protein groups were identified at a false discovery rate (FDR) of $1 \%$. On average, 5900 protein groups were quantified per sample (Figure 1B). Signal intensities for total quantified proteins spanned approximately seven orders of magnitudes (Figure 1C), with several well-known ovarian cancer markers, such as apolipoprotein A1 (APOA1), transthyretin (TTR), synuclein gamma (SNCG), stratifin (SFN), mesothelin (MSLN), DnaJ heat shock protein family (Hsp40) member A1 (DNAJA1), WAP four-disulfide core domain 2 (WFDC2), serine protease 8 (PRSS8), V-set domain containing T cell activation inhibitor 1 (VTCN1), insulin like growth factor binding protein 3 (IGFBP3), AT-rich interaction domain 1A (ARID1A), tumor protein p53 (TP53), vascular endothelial growth factor A (VEGFA), and notch receptor 1 (NOTCH1), being 
identified [13]. Pearson's correlation coefficients were correspondingly high at $0.82-0.84$ for inter- and intra-tissue replicates (Figure S2).

A
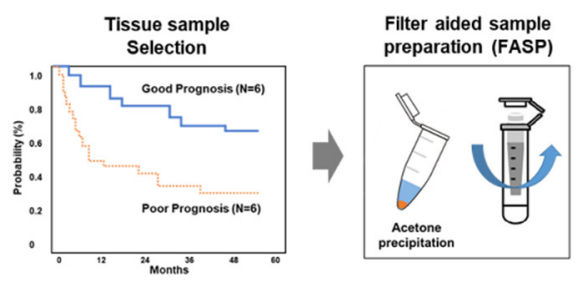

Feature selections
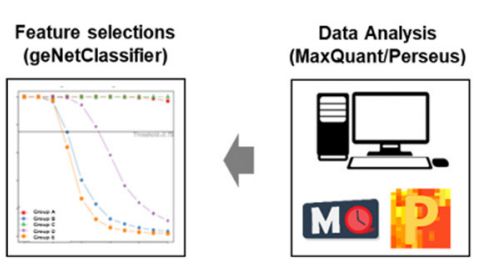
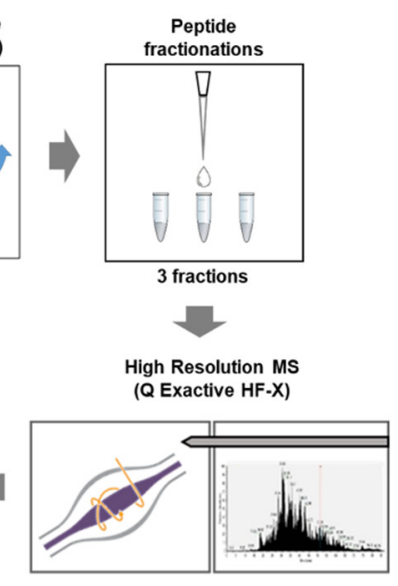

B

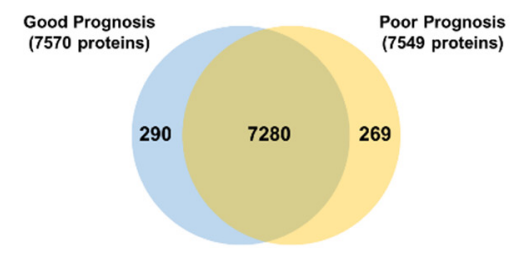

C

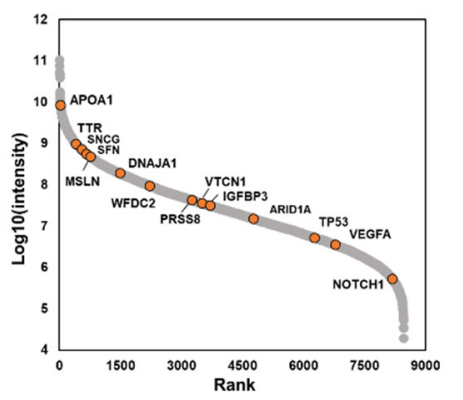

Figure 1. Proteomic analysis of ovarian cancer tissues with respect to survival outcome: (A) the protocol for proteomic analysis; (B) total number of proteins identified in each group of samples; (C) dynamic range of candidate biomarkers for high-grade serous ovarian carcinoma. MS, mass spectrometry.

On assessing tumor purity, using the ESTIMATE tool in the R package, mean ( \pm standard deviation) tumor purity score across the samples was $80.0( \pm 1.7)$ and $74.8( \pm 3.0)$ in the good and poor prognosis groups, respectively, indicating that the tumor purity was adequate to distinguish the tumor's signal from those of other cells (Figure S3) [14].

\subsubsection{Label-Free Quantification}

Furthermore, we compared the good and poor prognosis groups via principal component analysis (PCA) of a filtered list with approximately 6128 proteins (with $70 \%$ of valid intensity-based absolute quantification (iBAQ) values in at least one group). Although tumor proteomes correlated regardless of prognosis (Figure S2), the two groups were independently separated (Figure 2A).

To obtain functional insights into the proteomic data, we constructed a volcano plot to compare the expression levels between the good and poor prognosis groups. Pairwise comparisons via a $t$-test and filtering $(p<0.05$; fold-change, $>1.5$ ) revealed significant alterations in 658 proteins, of which 370 were upregulated and 288 were downregulated in the poor prognosis group (Figure $2 \mathrm{~B}$ and Table S3). Gene ontology (GO) enrichment analysis revealed that proteins upregulated in the good prognosis group were significantly enriched for terms such as "nucleobase-containing small molecule metabolism", "positive regulation of superoxide anion generation", "acute inflammatory response", "cellular component biogenesis", and "autophagy" (Figure 2C and Table S4). In contrast, proteins upregulated in the poor prognosis group were significantly enriched in "extracellular matrix organization", "wound healing", "muscle system process", "vesicle-mediated transport", "single-organism catabolism", and "antigen processing and presentation" (Figure 2D and Table S4). 
A

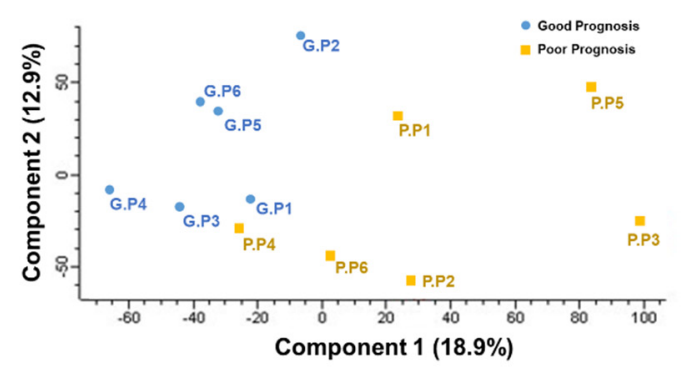

C

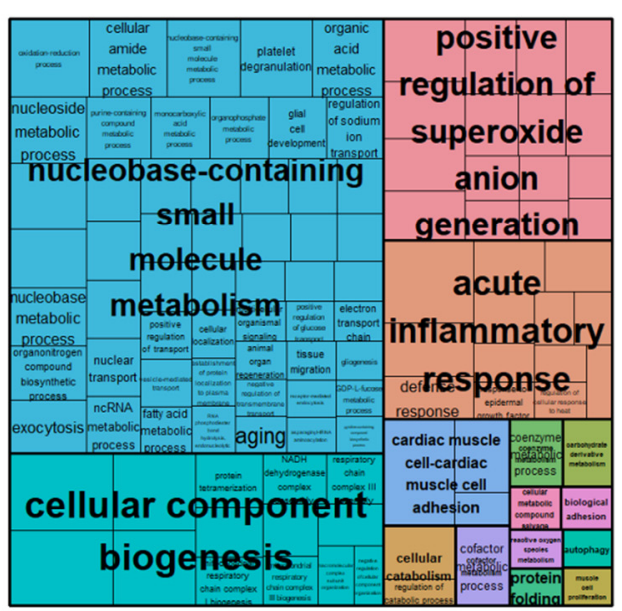

B

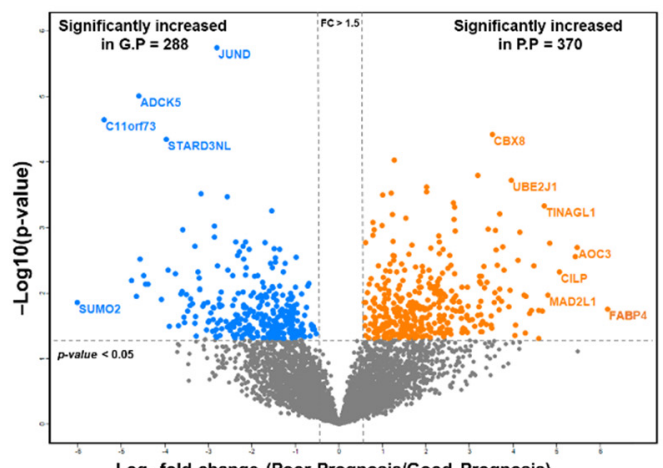

Log $_{2}$ fold-change (Poor Prognosis/Good Prognosis)

D

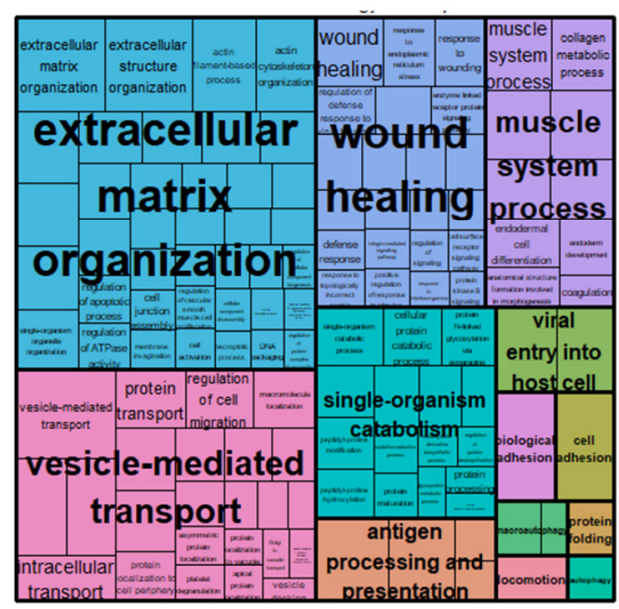

Figure 2. Statistical and functional differences between good and poor prognosis groups: (A) principal component analysis (PCA); (B) volcano plot; (C) gene ontology biological process (GOBP) enrichment tree-map of upregulated proteins in the good prognosis group; (D) GOBP enrichment tree-map of upregulated proteins in the poor prognosis group.

\subsubsection{Selection of Candidate Prognostic Biomarkers}

Further, we analyzed our proteomic data using the R/Bioconductor package "geNetClassifier (GNC)" [15] to rank proteins with the greatest discriminant power in an unbiased manner (Figure 3A). In total, 229 proteins exceeded the posterior probability cut-off $(>0.95)$ and were used in training the support vector machine (Table S5). The lowest error rate achieved by GNC was $0.08(8 \%)$ and corresponded to 41 proteins.

Among the top 20 proteins ranked through support-vector-machine analysis, 13 overlapped with significantly differentially expressed proteins (DEPs) (Figure 3B). After reviewing the data of each protein in the Human Protein Atlas database (https://www.proteinatlas.org) and evaluating the association between its expression and survival outcome in various malignancies, we selected phosphomevalonate kinase (PMVK), vascular adhesion protein 1 (VAP1), fatty acid-binding protein 4 (FABP4), and platelet factor 4 (PF4) as our biomarker candidates.

We further selected prognostic biomarker candidates on the basis of the following parameters: (1) significantly differentially expressed between the two groups, revealed through the $t$-test $(p<0.05)$; (2) quantification of expression in all samples; (3) presence of survival outcome data based on their expression in other malignancies; (4) availability of a commercial antibody; and (5) potential clinical utility (e.g., probability of being found in the blood). Thus, $\alpha 1$-antitrypsin (AAT), nuclear factor- $\mathrm{kB}$ (NFKB), APOA1, and $\alpha 1$-acid glycoprotein (AGP) were selected as additional biomarker candidates. 
A

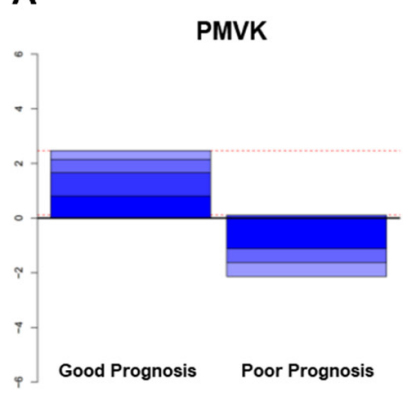

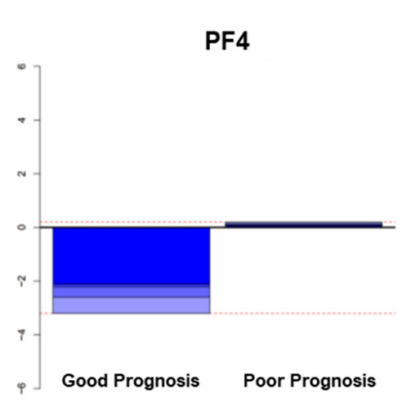

B

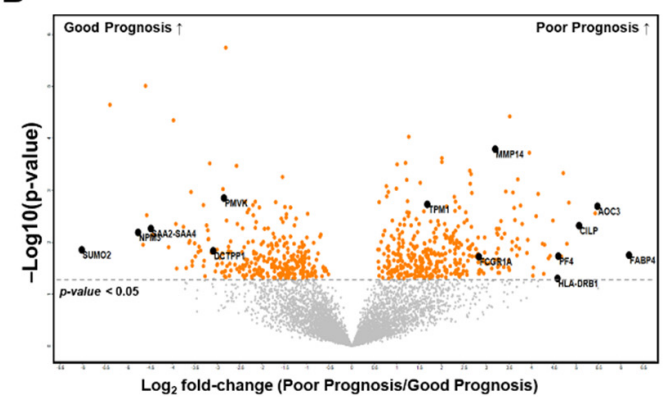

Figure 3. Selection of prognostic biomarker candidates using unbiased machine learning: (A) Discriminant power plots of the up-regulated gene (phosphomevalonate kinase, PMVK) in the good prognosis group and the up-regulated gene (platelet factor 4, PF4) in the poor prognosis group; (B) volcano plot with the 13 statistically significant differentially expressed proteins.

\subsection{Validation of Protein Biomarkers through IHC Analysis}

Prognostic validation of protein biomarkers was performed using tissue specimens obtained from 107 HGSOC patients, including the 12 patients whose tissue specimens were subjected to proteomic analysis. Clinicopathologic characteristics of 107 patients with HGSOC are presented in Table 1. The percentage of advanced-stage (FIGO III/IV) disease was 91.6\% (98/107). Of these 107 patients, 102 (95.3\%) underwent PDS, while 5 (4.7\%) underwent neoadjuvant chemotherapy (NAC) followed by interval debulking surgery. Optimal debulking (no gross residual tumor) was achieved in $68.2 \%$ (73/107). Germline BRCA1/2 mutations were observed in 50.5\% (54/107). Median length of observation was 23.7 months; during this observation, 51 patients (47.7\%) experienced relapse. The median PFS was 26.0 months, and the three-year PFS rate was $22.2 \%$.

Table 1. Clinicopathologic characteristics of the patients who underwent prognostic validation.

\begin{tabular}{cc}
\hline Characteristics & All $(\boldsymbol{n = 1 0 7 , \% )}$ \\
\hline Age, years & \\
Mean \pm SD & $55.6 \pm 10.1$ \\
Menopause & $69(71.9)$ \\
Personal history of breast cancer & $16(15.0)$ \\
Family history of breast cancer & $4(3.7)$ \\
Family history of ovarian cancer & $5(4.7)$ \\
Serum CA-125, IU/ml & \\
Median (range) & $677.5(5.1-11,630.0)$ \\
FIGO stage & \\
I-II & $9(8.4)$ \\
III & $68(63.6)$ \\
IV & $30(28.0)$ \\
PDS & $102(95.3)$ \\
NAC & $5(4.7)$ \\
Primary treatment strategy & $73(68.2)$ \\
Residual tumor after PDS/IDS & $21(19.6)$ \\
No gross & $7(6.5)$ \\
$<1$ cm & $6(5.6)$ \\
$\geq 1$ and <2 cm & \\
$\geq 2$ cm & $56(52.3)$ \\
Recurrence & $51(47.7)$ \\
No & $1(0.9)$ \\
Yes & $38(35.5)$ \\
PSR (within recurrent disease) & $12(11.2)$ \\
\hline No post-operative chemotherapy (within recurrent disease) &
\end{tabular}


Table 1. Cont.

\begin{tabular}{cc}
\hline Characteristics & All $(\boldsymbol{n}=\mathbf{1 0 7}, \mathbf{\%})$ \\
\hline Platinum sensitivity & \\
Platinum-sensitive $^{2}$ & $72(67.3)$ \\
Platinum-resistant & $12(11.2)$ \\
Germline BRCA mutation & \\
BRCA1 & $37(34.6)$ \\
BRCA2 & $17(15.9)$ \\
Both & 0
\end{tabular}

\begin{abstract}
${ }^{1}$ PSR was defined as relapse $\geq 6$ months after completion of taxane- and platinum-based chemotherapy, whereas PRR as relapse $<6$ months. ${ }^{2}$ In addition to PSR, the patients who completed taxane- and platinum-based chemotherapy and did not experience disease recurrence during at least six months of follow-up period were considered platinum-sensitive. Abbreviations: CA-125, cancer antigen 125; FIGO, International Federation of Gynecology and Obstetrics; IDS, interval debulking surgery; NAC, neoadjuvant chemotherapy; PDS, primary debulking surgery; PRR, platinum-resistant recurrence; PSR, platinum-sensitive recurrence; SD, standard deviation.
\end{abstract}

Herein, we used chemotherapy-naïve, formalin-fixed paraffin-embedded (FFPE) cancer tissues cut from the primary (non-metastatic) ovarian mass $(n=107)$. For the 12 patients, the specimens were the same as those used for proteomic analysis. Among the DEPs, AAT, NFKB, PMVK, VAP1, FABP4, PF4, APOA1, and AGP were subjected to further prognostic validation through IHC staining. Most tumor cells presented cytoplasmic and/or membranous staining patterns, except for VAP1, FABP4, PF4, and AGP, which occasionally displayed focal nuclear staining. APOA1 and AGP were also detected in the fibrotic stroma and inflammatory cells (Figure S4).

We then compared patient survival outcomes with respect to the expression levels of each protein and observed significant differences in PFS for the six proteins as follows: the group of patients with high expression levels of AAT, NFKB, and PMVK presented better PFS than those with low expression levels ( $p=0.024, p=0.009$, and $p=0.005$, respectively). For VAP1, FABP4, and PF4, the high expression group presented a reduced PFS relative to the low expression group $(p=0.010, p=0.010$, and $p=0.002$, respectively). However, the PFS did not significantly differ between patients with high and low expression levels of APOA1 and AGP, as determined through IHC staining (Figure 4). 

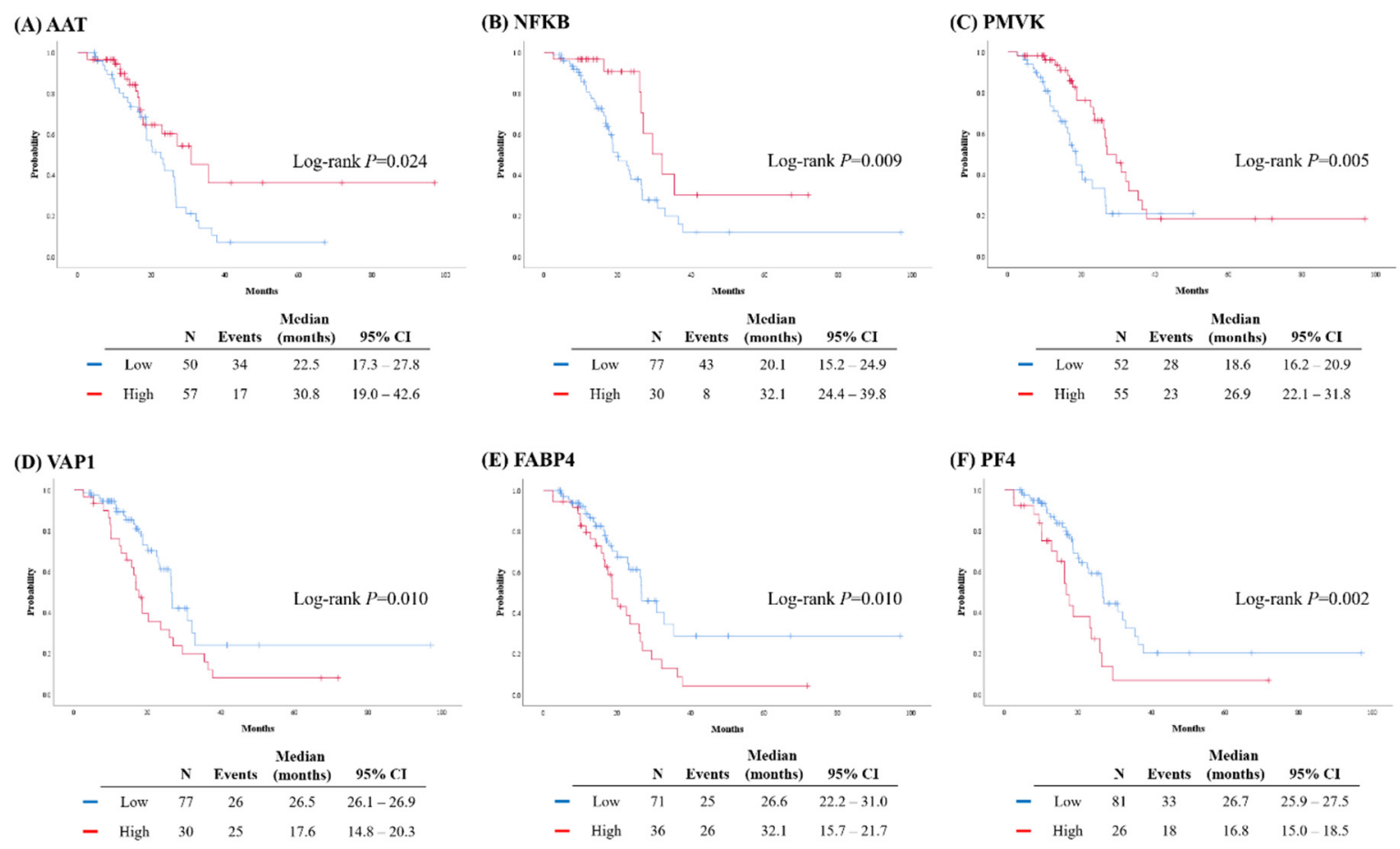

Figure 4. Comparison of progression-free survival (PFS) based on the expression levels of proteins: (A)

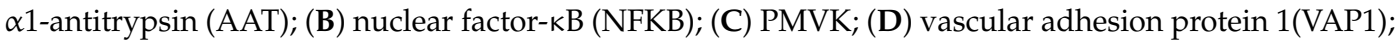
(E) fatty acid-binding protein 4 (FABP4); (F) PF4.

We further compared the clinicopathologic characteristics of patients with high and low expression levels of the six relevant protein biomarkers. No differences in expression levels were observed with respect to patient age ( $\geq 55$ vs. $<55$ years), FIGO stage (I-II vs. III-IV), or germline BRCA1/2 mutational status (mutation vs. wild-type) between the high and low expression groups. Patients with high NFKB expression levels achieved no gross residual tumor after surgery more commonly than those with low NFKB expression levels $(86.7 \%$ vs. $61.0 \% ; p=0.020)$. On assessing the platinum sensitivity of patients with respect to the expression profile of each protein, we observed a significant difference only for PVMK; patients with high PVMK expression levels were more sensitive to platinum-based chemotherapy than those with low PMVK expression levels (95.3\% vs. 75.6\%; $p=0.023$ ) (Table S6).

On multivariate analyses adjusting patient age at diagnosis, initial serum CA-125 level, FIGO stage, residual tumor after surgery, and germline BRCA1/2 mutational status, high expression of AAT, NFKB, and PMVK were identified as independent favorable prognostic biomarkers for PFS (AAT (adjusted hazard ratio (aHR), 0.398; 95\% confidence interval (CI), 0.207-0.768; $p=0.006)$, NFKB (aHR, 0.424; 95\% CI, 0.196-0.920; $p=0.030$ ), and PMVK (aHR, 0.430; 95\% CI, 0.228-0.809; $p=0.009)$ ). In contrast, high expression of VAP1, FABP4, and PF4 were considered independent poor prognostic biomarkers for PFS (VAP1 (aHR, 1.911; 95\% CI, 1.089-3.354; $p=0.024)$, FABP4 (aHR, 1.908; 95\% CI, 1.093-3.331; $p=0.023)$, and PF4 (aHR, 2.071; 95\% CI, 1.139-3.765; $p=0.017)$ ) (Table 2). 
Table 2. Factors associated with progression-free survival.

\begin{tabular}{|c|c|c|c|c|c|c|c|c|c|c|c|c|}
\hline \multirow{2}{*}{ Characteristics } & \multicolumn{12}{|c|}{ Multivariate Analysis } \\
\hline & aHR & $95 \% \mathrm{CI}$ & aHR & $95 \% \mathrm{CI}$ & aHR & $95 \% \mathrm{CI}$ & aHR & $95 \% \mathrm{CI}$ & aHR & $95 \% \mathrm{CI}$ & aHR & $95 \% \mathrm{CI}$ \\
\hline Age, years & & $p=0.072$ & & $p=0.667$ & & $p=0.879$ & & $p=0.417$ & & $p=0.332$ & & $p=0.365$ \\
\hline$\geq 55$ vs. $<55$ & 1.755 & $0.951-3.238$ & 1.133 & $0.641-2.005$ & 1.046 & $0.586-1.866$ & 1.265 & $0.717-2.230$ & 1.327 & $0.750-2.349$ & 1.300 & $0.737-2.295$ \\
\hline CA-125, IU/ml & & $p=0.028$ & & $p=0.061$ & & $p=0.103$ & & $p=0.066$ & & $p=0.124$ & & $p=0.157$ \\
\hline$\geq 700$ vs. $<700$ & 1.911 & $1.073-3.405$ & 1.720 & $0.976-3.031$ & 1.603 & $0.909-2.826$ & 1.695 & $0.965-2.977$ & 1.553 & $0.886-2.721$ & 1.500 & $0.856-2.628$ \\
\hline FIGO stage & & $p=0.470$ & & $p=0.182$ & & $p=0.260$ & & $p=0.304$ & & $p=0.281$ & & $p=0.255$ \\
\hline III-IV vs. I-II & 2.149 & $0.270-17.098$ & 4.010 & $0.522-30.829$ & 3.220 & $0.421-24.652$ & 2.920 & $0.379-22.499$ & 3.066 & $0.400-23.498$ & 3.227 & $0.429-24.274$ \\
\hline Residual tumor after PDS/IDS & & $p=0.057$ & & $p=0.183$ & & $p=0.019$ & & $p=0.142$ & & $p=0.118$ & & $p=0.137$ \\
\hline Gross vs. No gross & 1.732 & $0.985-3.048$ & 1.474 & $0.833-2.608$ & 2.020 & $1.124-3.630$ & 1.531 & $0.868-2.703$ & 1.578 & $0.891-2.794$ & 1.538 & $0.872-2.711$ \\
\hline Germline $B R C A$ status & & $p=0.085$ & & $p=0.101$ & & $p=0.425$ & & $p=0.094$ & & $p=0.088$ & & $p=0.162$ \\
\hline $\begin{array}{c}\text { Mutation vs. WT } \\
\text { AAT }\end{array}$ & 0.598 & $\begin{array}{c}0.333-1.073 \\
p=0.006\end{array}$ & 0.614 & $0.343-1.099$ & 0.780 & $0.424-1.436$ & 0.600 & $0.329-1.091$ & 0.594 & $0.326-1.081$ & 0.654 & $0.361-1.186$ \\
\hline High vs. Low & 0.398 & $0.207-0.768$ & & & & & & & & & & \\
\hline NFKB & & & & $p=0.030$ & & & & & & & & \\
\hline High vs. Low & & & 0.424 & $0.196-0.920$ & & & & & & & & \\
\hline PMVK & & & & & & $p=0.009$ & & & & & & \\
\hline High vs. Low & & & & & 0.430 & $0.228-0.809$ & & & & & & \\
\hline VAP1 & & & & & & & & $p=0.024$ & & & & \\
\hline High vs. Low & & & & & & & 1.911 & $1.089-3.354$ & & & & \\
\hline FABP4 & & & & & & & & & & $p=0.023$ & & \\
\hline High vs. Low & & & & & & & & & 1.908 & $1.093-3.331$ & & \\
\hline PF4 & & & & & & & & & & & & $p=0.017$ \\
\hline High vs. Low & & & & & & & & & & & 2.071 & $1.139-3.765$ \\
\hline
\end{tabular}

Abbreviations: aHR, adjusted hazard ratio; CI, confidence interval; CA-125, cancer antigen 125; FIGO, International Federation of Gynecology and Obstetrics; IDS, interval debulking surgery; PDS, primary debulking surgery; WT, wild-type; AAT, $\alpha 1$-antitrypsin; NFKB, nuclear factor-kB; PMVK, phosphomevalonate kinase; VAP1, vascular adhesion protein 1; FABP4, fatty acid-binding protein 4 ; PF4, platelet factor 4. 
Furthermore, we constructed models predicting 18-month PFS by combining clinical variables and IHC results. Here, we considered two types of models: regression- and score-based. The performance of predictive models was evaluated via the leave-one-out cross validation method for the regression-based models. The best model included the following variables: initial serum CA-125 levels ( $\geq 700$ vs. $<700$ $\mathrm{IU} / \mathrm{mL}$ ), germline $B R C A 1 / 2$ mutational status (mutation vs. wild-type), residual tumor after surgery (gross vs. no gross), FIGO stage (III-IV vs. I-II), and expression levels (high or low) of the six protein biomarkers on IHC staining of ovarian cancer tissue. For the regression-based model, the estimated areas under the receiver operating characteristic curves (AUCs) from the training and test datasets were 0.898 and 0.776 , respectively. In the score-based model, the scores of each predictor $(0$ or 1$)$ were added to give a total score. Samples with the total score $\geq 7$ were classified as "high-risk", and showed an AUC of 0.855. Both regression- and score-based models displayed better performance than those comprising only clinical variables (Table S7).

\section{Discussion}

In the current study, we performed label-free liquid chromatography-mass spectrometry (LC-MS/MS)-based proteomic analysis on chemotherapy-naïve, fresh-frozen primary HGSOC tissues. Upon validation with complementary IHC staining for FFPE HGSOC tissue specimens, we identified six protein biomarkers to predict the prognosis of HGSOC; expression levels of AAT, NFKB, PMVK, VAP1, FABP4, and PF4 in ovarian cancer tissue were associated with PFS.

AAT, encoded by SERPINA1 in humans, is a serine protease inhibitor that influences tumor behavior depending on the context and/or cancer type. Consistent with our results, enrichment of SERPINA1 mRNA was associated with a good prognosis in HGSOC [16]; however, no association was observed between survival of patients with HGSOC and AAT expression levels, as assessed via IHC staining [17]. Two previous studies evaluated serum AAT levels in patients with epithelial ovarian cancer and reported that AAT may contribute to a differential diagnosis and help predict chemoresistance $[18,19]$.

NFKB is constitutively activated in several cancers. The p100 subunit of NFKB, a precursor of the active $\mathrm{p} 52$ subunit, has been suggested to counteract the tumorigenic effects of p52 in breast cancer [20]. In ovarian cancer, NFKB p52 promoted cancer progression, resulting in an unfavorable prognosis [21]. To our knowledge, our study is the first to report the association between high expression of NFKB p100 and improved PFS in patients with HGSOC.

Furthermore, this study shows that PMVK, an enzyme involved in cholesterol synthesis and lipid metabolism, can be considered a novel prognostic biomarker for HGSOC. In estrogen receptor-positive breast cancer, high expression of $P M V K$ gene was positively associated with responses to chemotherapeutic agents [22]. Similarly, herein, high expression of PMVK, assessed via IHC staining, was significantly associated with platinum sensitivity and improved the survival of patients with HGSOC.

VAP1, an adhesion molecule mediating interactions between various inflammatory and endothelial cells, may be associated with tumor invasion and metastasis. High expression of VAP1 was associated with a lower overall survival in breast cancer [23,24]. In our study, VAP was identified as a poor prognostic factor for PFS in patients with HGSOC.

A previous study reported that FABP4 promotes HGSOC progression by mediating lipid metabolism in cancer cells [25]. Furthermore, the present results indicate that high expression of FABP4, assessed via IHC staining, was associated with a poor PFS.

PF4 is a platelet-activating chemokine that induces thrombocytosis and thromboembolism. High serum PF4 levels were associated with poor survival and an increased risk of venous thromboembolism in patients with pancreatic adenocarcinoma [26]. According to a microarray study using 51 HGSOC samples, increased expression of PF4 mRNA was negatively associated with patients' overall survival [27]. Similarly, our study results also indicate that high expression of PF4, assessed via IHC staining, was associated with a reduced PFS. 
APOA1 and AGP are acute-phase reactants, and their serum levels have been assessed in epithelial ovarian cancer to assess their potential as diagnostic or prognostic predictive biomarkers [28-31]. However, tissue expression and clinical implications of APOA1 and AGP in HGSOC have not been determined. In the current study, we revealed that expressions of APOA1 and AGP in HGSOC cancer tissues were not associated with patient survival outcomes. Furthermore, in several tissue microarray (TMA) cores, APOA1 and AGP were expressed strongly only in the stroma and inflammatory cells, but not in cancer cells, suggesting the host immune response to cancer.

A potential limitation of our study is that the menopausal status may have influenced the expression levels of AAT, NFKB, PMVK, VAP1, FABP4, and PF4. However, during the proteomic analysis, the proportion of menopausal women was the same between the good and poor prognosis groups (66.7\%). Moreover, during subsequent IHC analysis for prognostic validation, the patients' ages were adjusted for multivariate analyses to identify the prognostic factors for PFS.

Since the CPTAC presented a proteomic landscape of 169 HGSOC tumors [10], several studies have focused on HGSOC using MS-based proteomics. Coscia et al. performed integrative proteomic profiling of ovarian cancer cell lines and HGSOC tumors and revealed two distinct clusters, epithelial and mesenchymal, which displayed different clinical outcomes [11]. Dieters-Castator et al. performed label-free quantitative proteomic analysis using 10 fresh-frozen HGSOC tissues and 10 fresh-frozen endometrioid carcinoma tissues, and identified diagnostic biomarkers specific to endometrioid carcinoma. Furthermore, the eight-marker panel, generated in that study, showed good performance in discriminating endometrioid carcinoma from HGSOC [12].

Unlike previous studies focused on a differential diagnosis or clustering of HGSOC, this study aimed to investigate protein biomarkers predicting survival outcomes. IHC staining on FFPE HGSOC tissue specimens revealed that expression levels of the six protein biomarkers were not different between the early-stage and advanced-stage disease, and between diseases with high and low initial serum CA-125 levels. Nevertheless, our results indicate that high expressions of AAT, NFKB, and PMVK are favorable prognostic biomarkers for PFS, whereas high expressions of VAP1, FABP4, and PF4 are poor prognostic biomarkers for PFS. These six protein biomarkers, along with well-known prognostic factors including the stage and size of residual tumors, are expected to increase the accuracy of predicting relapse after primary treatment. Such improvements in prognostic prediction would facilitate the development of individualized therapies. For instance, if a patient is identified as being at high risk for recurrence, she may receive intraperitoneal chemotherapy or bevacizumab maintenance therapy in addition to the standard treatment and would be placed on a frequent surveillance schedule for earlier detection of relapse. Moreover, each of the six protein biomarkers, identified in our present study, can be considered a target for novel molecular therapeutic agents against HGSOC. However, further translational studies using cell lines and cancer tissues are essential for assessing biological effectiveness of the protein biomarkers and for identifying relevant pathways.

Herein, we performed IHC staining to validate candidate prognostic protein biomarkers. IHC is more cost-effective and simpler to use in the clinical setting than DNA or RNA PCRs and exome- or transcriptome-level next-generation sequencing methods. IHC can be utilized for prognosis prediction immediately during pathologic examination of the tissue obtained during ovarian cancer surgery.

For further studies validating a predictive model consisting of all the six protein biomarkers, we calculated the estimated powers for various sample sizes and censoring rates, which were drawn from a simple simulation study ( $p<0.05 ; 1000$ replicates), using the estimates from our multivariate model and the predictor frequencies from our dataset (Figure S5).

This study has several limitations. First, owing to the retrospective study design, issues such as selection bias might exist. Second, the sample size used in our study may be insufficient for further discovery and validation of protein biomarkers. Third, external validation of our study results is necessary. Fourth, additional studies, elucidating the mechanisms of action of each protein biomarker, were not performed herein. Lastly, we did not evaluate interactions between among these protein biomarkers. Despite these limitations, we faithfully applied a two-step approach consisting 
of proteomic and bioinformatic analyses and subsequent IHC staining for HGSOC tissue to identify prognostic protein biomarkers. Moreover, we developed predictive models comprising the six protein biomarkers and clinical variables for 18-month PFS of HGSOC patients. Such models displayed better prediction potential than those comprising only clinical variables. Especially, the proposed predictive model was further simplified as a score-based model, which provides comparable performance and substantial intuitiveness.

\section{Materials and Methods}

This retrospective study was approved by the Institutional Review Board of Seoul National University Hospital (SNUH; No. C-1712-083-907), and conducted in accordance with the Declaration of Helsinki.

\subsection{Study Design}

This study included two steps: (1) proteomic and bioinformatic analysis for biomarker discovery; and (2) IHC staining for prognostic validation of candidate biomarkers (Figure S6).

In the first step, we used fresh-frozen primary (non-metastatic) ovarian cancer tissues obtained intraoperatively and stored at the SNUH Human Biobank for research purposes. We identified patients who met the following inclusion criteria: (1) older than 18 years; (2) diagnosed with HGSOC between June 2012 and December 2016; (3) underwent PDS; and (4) agreed to donate biospecimens and provide written informed consent. Patients with any malignancy other than ovarian cancer, those who received NAC, those with insufficient clinical data or those lost to follow-up, or those with severe co-morbidities were excluded. On the basis of their PFS, patients were divided into the favorable (good) prognosis group ( $\geq 18$ months) and poor prognosis group ( $<18$ months). In total, 12 patients from the two groups ( 6 for each group) were selected for further proteomic analysis, and proteomic profiles were compared between the two groups.

In the second step, we used FFPE primary (non-metastatic) ovarian cancer tissues stored in the pathology archive of SNUH. In contrast with the first step, we identified patients meeting the following inclusion criteria: (1) older than 18 years; (2) diagnosed with HGSOC between June 2012 and December 2018; (3) whose ovarian cancer tissue was obtained during chemotherapy-naïve status (such as at the time of PDS, or during diagnostic laparoscopy in case of NAC); and (4) agreed to donate their pathologic specimens for research purposes and provided written informed consent. Patients with insufficient clinical data or those lost to follow-up or those with severe co-morbidities were excluded. In total, 107 patients with primary HGSOC were included in this step.

\subsection{Proteomic and Bioinformatic Analyses}

\subsubsection{Tissue Preparation}

Tissue samples were prepared using filter-aided sample preparation (FASP), as previously described [32]. Briefly, frozen tissue samples were homogenized using lysis buffer ( $4 \%$ sodium dodecyl sulfate (SDS), $2 \mathrm{mM}$ Tris(2-carboxyethyl)phosphine (TCEP), and $0.1 \mathrm{M}$ Tris- $\mathrm{HCl} \mathrm{pH} \mathrm{7.4),} \mathrm{and} \mathrm{protein}$ concentration was determined using a reducing agent-compatible bicinchoninic acid (BCA) protein assay kit (Thermo Fisher Scientific, Waltham, MA, USA) in accordance with the manufacturer's instructions. To eliminate contaminants, we performed acetone precipitation using $250 \mu \mathrm{g}$ of the lysate at $-20^{\circ} \mathrm{C}$. Each protein pellet was dissolved in $50 \mu \mathrm{L}$ reduction buffer $(4 \% \mathrm{SDS}, 0.1 \mathrm{mM}$ dithiothreitol (DTT), and $0.1 \mathrm{M}$ Tris-HCl, $\mathrm{pH}$ 7.4) and heated at $95^{\circ} \mathrm{C}$ for $15 \mathrm{~min}$. The reduced proteins were loaded onto a $30 \mathrm{~K}$ spin filter (Millipore, Billerica, MA, USA), and buffer was exchanged for UA solution (8 M urea in $0.1 \mathrm{M}$ Tris- $\mathrm{HCl}, \mathrm{pH}$ 8.5) via centrifugation. After triple UA exchanging, the reduced cysteines were alkylated with $0.05 \mathrm{M}$ iodoacetamide (IAA) in UA solution for $30 \mathrm{~min}$ at ambient temperature in the dark. Thereafter, UA buffer was exchanged for $40 \mathrm{mM}$ ammonium bicarbonate (ABC), and the samples were digested with trypsin (enzyme to substrate ratio of $1: 100$ ) at $37^{\circ} \mathrm{C}$ for $16 \mathrm{~h}$. Further, the 
digested peptides were harvested via centrifugation, and an additional elution step was performed using $40 \mathrm{mM} \mathrm{ABC}$ and $0.5 \mathrm{M} \mathrm{NaCl}$.

\subsubsection{Desalting and Peptide Fractionation of Individual Samples}

Peptide concentrations were measured using the tryptophan fluorescence (WF) assay, as previously described [33]. Digested peptides $(20 \mu \mathrm{g})$ were acidified with trifluoroacetic acid (TFA) and then loaded directly onto house-made Stage-Tip with polystyrenedivinylbenzene-reversed phase sulfonate (SDB-RPS) material [34]. StageTip was washed thrice with $100 \mu \mathrm{L} 0.2 \%$ TFA. Three fractionations were performed using elution buffer 1, 2, and 3. All eluted peptides were dried in a SpeedVac centrifuge.

\subsubsection{Offline High-pH Reversed-Peptide Fractionation for Library Construction}

For library construction, pooled peptides were fractionated via high $\mathrm{pH}$ reversed-phase liquid chromatography (RPLC) using an Agilent 1290 bioinert high performance liquid chromatography (HPLC) (Agilent, Santa Clara, CA, USA) equipped with an analytical column $(4.6 \times 250 \mathrm{~mm}, 5 \mu \mathrm{m})$, as previously described [32]. Solvent A consisted of $15 \mathrm{mM}$ ammonium hydroxide in water, and solvent B consisted of $15 \mathrm{mM}$ ammonium hydroxide in $90 \%$ acetonitrile (ACN). The peptides were separated along a gradient of $5 \%-35 \% \mathrm{ACN}$ at a flow rate of $0.2 \mathrm{~mL} / \mathrm{min}$. In total, 96 fractions were concatenated to mix different parts of the gradient into 24 fractions. The fractions were lyophilized and stored at $-80^{\circ} \mathrm{C}$ until MS analysis.

\subsubsection{LC-MS/MS Analysis}

All LC-MS/MS analyses were conducted using an Ultimate 3000 UHPLC system (Dionex, Sunnyvale, CA, USA) coupled with Q-Exactive HF-X mass spectrometry (Thermo Fisher Scientific, Waltham, MA, USA), as previously described with some modifications [32]. Peptides were separated on a two-column system equipped with a trap column $(300 \mu \mathrm{m} \times 5 \mathrm{~mm})$ and an analytic column $(75 \mu \mathrm{m} \times$ $50 \mathrm{~cm}$ ), using $90-\mathrm{min}$ gradients from $7 \%$ to $32 \% \mathrm{ACN}$ at a flow rate of $300 \mathrm{nl} / \mathrm{min}$. Column temperature was maintained at $60^{\circ} \mathrm{C}$ using a column heater. For label-free quantification using the data-dependent acquisition (DDA) method, a survey scan (350 to $1650 \mathrm{~m} / \mathrm{z}$ ) was acquired with a resolution of 70,000 at $\mathrm{m} / \mathrm{z} 200$. A top-15 method was used to select the precursor ion with an isolation window of $1.2 \mathrm{~m} / \mathrm{z}$. MS/MS spectra were acquired at a higher-energy collisional dissociation (HCD)-normalized collision energy (NCE) of 30 with a resolution of 17,500 at $\mathrm{m} / \mathrm{z} 200$. Maximum ion injection durations for the full and MS/MS scans were 20 and $100 \mathrm{~ms}$, respectively.

\subsubsection{Data Processing}

All MS raw files were processed using MaxQuant (version 1.6.1.0) [35]. MS/MS spectra were searched against the Human Uniprot protein sequence database (December 2014 with 88,657 entries) using the Andromeda search engine [36]. Primary searches were performed using a 6 ppm precursor ion tolerance for total protein-level analysis. MS/MS ion tolerance was set to $20 \mathrm{ppm}$. Cysteine carbamidomethylation was set as a fixed modification. Protein $\mathrm{N}$-acetylation and methionine oxidation were considered variable modifications. Enzyme specificity was set to full tryptic digestion. Peptides with a minimum length of six amino acids and up to two missed cleavages were considered. The required FDR was set to $1 \%$ at peptide, protein, and modification levels. To maximize the number of quantification events across samples, we enabled the "Match between Runs" option on the MaxQuant platform.

\subsubsection{Label-Free Quantification and Statistical Analysis}

For label-free quantification, the iBAQ algorithm was used as part of the MaxQuant platform [37]. Briefly, iBAQ values, determined using MaxQuant, were the raw intensities divided by the number of theoretical peptides. Thus, iBAQ values were proportional to molar quantities of the proteins. Perseus software was used for statistical analysis [38]. First, we eliminated proteins identified as "reverse" 
and "only identified by site". After filtering values of at least 70\% in each group, missing values were imputed using a width of 0.3 and down shift of 1.8. Finally, data were normalized using a width adjustment function, which subtracts the medians and scales all values in a sample to yield equal interquartile ranges (IQRs) [39]. For pairwise proteome comparisons, we performed a two-sided $t$-test with a significance level ( $p$-value) of $<0.05$ and fold-change of $>1.5$. Support vector machine analysis was performed using the R/Bioconductor package "GNC" [15].

\subsubsection{Bioinformatic Analysis}

GO enrichment analysis was performed using the DAVID bioinformatics resources (https://david. ncifcrf.gov/). GO-terms and corresponding $p$-values were subsequently submitted to ReViGO [40], and visualized using high semantic similarity-based treemaps. Tumor purity was assessed using the $R$ package "ESTIMATE" on the basis of the expression levels of marker genes in stromal and immune cells [41].

\subsection{Validation via IHC Analysis}

\subsubsection{TMA Construction}

Prognostic implications of protein biomarkers, identified through proteomic analyses, were validated via IHC staining using a separate dataset consisting of chemotherapy-naïve, FFPE cancer tissues resected from the primary (non-metastatic) ovarian mass intraoperatively during debulking surgery (PDS cases) or diagnostic laparoscopy (NAC cases) $(n=107)$. After tissues were retrieved from the pathology archive of SNUH, they were histologically assessed through hematoxylin and eosin staining. To construct a TMA, three cores ( $2 \mathrm{~mm}$ in diameter) per patient were embedded in new recipient FFPE blocks using a trephine apparatus (Superbiochips Laboratories, Seoul, Korea).

\subsubsection{IHC Staining}

IHC staining for AAT, NFKB, PMVK, VAP1, FABP4, PF4, APOA1, and AGP was performed using $4 \mu \mathrm{m}$ thick TMA sections using a Benchmark autostainer (Ventana, Tucson, AZ, USA) in accordance with the manufacturer's instructions (Table S8).

Because IHC staining of these eight antibodies and its prognostic effects was not previously evaluated in HGSOC, we determined the optimal cutoff values for each IHC staining, based on the sample distribution and prognostic significance (Table S8). Briefly, the extent (0-20\%, 20-50\%, 50-70\%, 70-100\%) and intensity (absent, weak, moderate, strong) of cytoplasmic/membranous immunoreactivity were semi-quantitatively assessed. Thereafter, the expression level of each protein was dichotomized into high versus low expression (Figure S4).

\subsection{Statistical Analysis}

Descriptive statistics were used to describe clinicopathologic characteristics of the study population. Patient characteristics were compared between the good and poor prognosis groups, and between groups showing low and high expression of each protein biomarker. We used Student's $t$ and Mann-Whitney U tests to compare continuous variables, and Pearson's Chi-squared and Fisher's exact tests to compare categorical variables. Kaplan-Meier methods with log-rank test were used for survival analysis. Multivariate analysis was performed using a Cox proportional-hazards model, and aHRs and 95\% CIs were calculated. These analyses were conducted using SPSS software (version 25.0; SPSS Inc., Chicago, IL, USA). All statistical tests were two-sided, and a $p$-value $<0.05$ was considered statistically significant.

We constructed regression- and score-based models predicting 18-month PFS using clinical variables and IHC results of 107 patients with primary HGSOC. To evaluate the performance of regression-based predictive models, we performed leave-one-out cross-validation with the consideration of a small sample size. In brief, leave-one-out cross-validation constructs $n$ models 
repetitively, by training the model with $n-1$ samples and testing with the remaining one, where $n$ is the sample size. This analysis was repeated for all samples, and $n$ predicted values were obtained on the basis of $n$ models. We computed AUC using the predicted values and the observed values of the response variable. Finally, we simplified the regression-based model into a score-based model. In this study, each predictor has a single binary value, either 0 or 1 . We inverted the original values of the predictors with a negative coefficient $(0 / 1$ to $1 / 0)$ so that all the direction of effects be positive. Then, a total score for the prediction of 18-month PFS was determined by simply adding all the predictors without coefficient.

\section{Conclusions}

In conclusion, we successfully generated a proteomic landscape of HGSOC and identified six protein biomarkers to predict the prognosis of HGSOC. These biomarkers are potentially applicable for the development of novel molecular therapeutic agents in the future. Further translational studies and prospective validation studies are warranted to determine the underlying mechanisms of action and interactions among these biomarkers.

Supplementary Materials: The following are available online at http://www.mdpi.com/2072-6694/12/4/790/s1, Figure S1: A comparison of progression-free survival between good and poor prognosis groups; Figure S2: Reproducibility between protein expression values obtained from the good and poor prognosis groups; Figure S3: Assessment of tumor purity in the good and poor prognosis groups; Figure S4: Representative images of high expression and low expression of each protein. (a) AAT; (b) NFKB; (c) PMVK; (d) VAP1; (e) FABP4; (f) PF4; (g) APOA1; (h) AGP; Figure S5: Power analysis of a multivariate model consisting of the six protein biomarkers with variable sample sizes and censoring rates; Figure S6: Flow diagram illustrating the overall study design; Table S1: Clinicopathologic characteristics of the patients who underwent proteomic analysis; Table S2: List of total identified proteins; Table S3: List of differentially expressed proteins between good and poor prognosis groups; Table S4: Results of gene ontology enrichment in differentially expressed proteins; Table S5: Ranked protein list obtained using "geNetClassifier"; Table S6: Comparisons of patients' clinicopathologic characteristics by immunohistochemical staining results; Table S7: Comparisons of performance among the developed models predicting 18-month progression-free survival; Table S8: Information on antibodies used in this study and interpretation of results obtained via immunohistochemical staining.

Author Contributions: Conceptualization, D.H. and M.L.; Formal analysis, S.I.K., M.J., K.D., S.L., and D.H.; Investigation, S.I.K., M.J., K.D., S.L., C.L., D.H., and M.L.; Project administration, M.L.; Supervision, D.H. and M.L.; Writing—original draft, S.I.K., M.J., and M.L.; Writing—review \& editing, all authors. All authors have read and agreed to the published version of the manuscript.

Funding: This research was supported by a grant of the Korea Health Technology R\&D Project through the Korea Health Industry Development Institute (KHIDI), funded by the Ministry of Health \& Welfare, Korea (No. HI19C0664). This work was also funded by grants from the Seoul National University Hospital Research Fund (No. 0320170220 and 0320190260).

Acknowledgments: The biospecimens for this study were provided by the Seoul National University Hospital Human Biobank, a member of the National Biobank of Korea, which is supported by the Ministry of Health and Welfare. All samples, derived from the National Biobank of Korea, were obtained with informed consent under institutional review board-approved protocols.

Conflicts of Interest: The authors declare no conflict of interest.

\section{References}

1. Bray, F.; Ferlay, J.; Soerjomataram, I.; Siegel, R.L.; Torre, L.A.; Jemal, A. Global cancer statistics 2018: GLOBOCAN estimates of incidence and mortality worldwide for 36 cancers in 185 countries. CA Cancer J. Clin. 2018, 68, 394-424. [CrossRef] [PubMed]

2. Siegel, R.L.; Miller, K.D.; Jemal, A. Cancer statistics, 2019. CA Cancer J. Clin. 2019, 69, 7-34. [CrossRef] [PubMed]

3. Cho, K.R.; Shih Ie, M. Ovarian cancer. Annu. Rev. Pathol. 2009, 4, 287-313. [CrossRef] [PubMed]

4. Bristow, R.E.; Tomacruz, R.S.; Armstrong, D.K.; Trimble, E.L.; Montz, F.J. Survival effect of maximal cytoreductive surgery for advanced ovarian carcinoma during the platinum era: A meta-analysis. J. Clin. Oncol. 2002, 20, 1248-1259. [CrossRef] 
5. Chi, D.S.; Franklin, C.C.; Levine, D.A.; Akselrod, F.; Sabbatini, P.; Jarnagin, W.R.; DeMatteo, R.; Poynor, E.A.; Abu-Rustum, N.R.; Barakat, R.R. Improved optimal cytoreduction rates for stages IIIC and IV epithelial ovarian, fallopian tube, and primary peritoneal cancer: A change in surgical approach. Gynecol. Oncol. 2004, 94, 650-654. [CrossRef] [PubMed]

6. Cannistra, S.A. Cancer of the ovary. N. Engl. J. Med. 2004, 351, 2519-2529. [CrossRef]

7. Zhang, B.; Wang, J.; Wang, X.; Zhu, J.; Liu, Q.; Shi, Z.; Chambers, M.C.; Zimmerman, L.J.; Shaddox, K.F.; Kim, S.; et al. Proteogenomic characterization of human colon and rectal cancer. Nature 2014, 513, $382-387$. [CrossRef]

8. Mertins, P.; Mani, D.R.; Ruggles, K.V.; Gillette, M.A.; Clauser, K.R.; Wang, P.; Wang, X.; Qiao, J.W.; Cao, S.; Petralia, F.; et al. Proteogenomics connects somatic mutations to signalling in breast cancer. Nature 2016, 534, 55-62. [CrossRef]

9. Okayama, A.; Kimura, Y.; Miyagi, Y.; Oshima, T.; Oshita, F.; Ito, H.; Nakayama, H.; Nagashima, T.; Rino, Y.; Masuda, M.; et al. Relationship between phosphorylation of sperm-specific antigen and prognosis of lung adenocarcinoma. J. Proteomics 2016, 139, 60-66. [CrossRef]

10. Zhang, H.; Liu, T.; Zhang, Z.; Payne, S.H.; Zhang, B.; McDermott, J.E.; Zhou, J.Y.; Petyuk, V.A.; Chen, L.; Ray, D.; et al. Integrated Proteogenomic Characterization of Human High-Grade Serous Ovarian Cancer. Cell 2016, 166, 755-765. [CrossRef]

11. Coscia, F.; Watters, K.M.; Curtis, M.; Eckert, M.A.; Chiang, C.Y.; Tyanova, S.; Montag, A.; Lastra, R.R.; Lengyel, E.; Mann, M. Integrative proteomic profiling of ovarian cancer cell lines reveals precursor cell associated proteins and functional status. Nat. Commun. 2016, 7, 12645. [CrossRef]

12. Dieters-Castator, D.Z.; Rambau, P.F.; Kelemen, L.E.; Siegers, G.M.; Lajoie, G.A.; Postovit, L.M.; Kobel, M. Proteomics-Derived Biomarker Panel Improves Diagnostic Precision to Classify Endometrioid and High-grade Serous Ovarian Carcinoma. Clin. Cancer Res. 2019, 25, 4309-4319. [CrossRef] [PubMed]

13. Nolen, B.M.; Lokshin, A.E. Protein biomarkers of ovarian cancer: The forest and the trees. Future Oncol. 2012, 8, 55-71. [CrossRef] [PubMed]

14. Aran, D.; Sirota, M.; Butte, A.J. Systematic pan-cancer analysis of tumour purity. Nat. Commun. 2015, 6, 8971. [CrossRef] [PubMed]

15. Aibar, S.; Fontanillo, C.; Droste, C.; Roson-Burgo, B.; Campos-Laborie, F.J.; Hernandez-Rivas, J.M.; De Las Rivas, J. Analyse multiple disease subtypes and build associated gene networks using genome-wide expression profiles. BMC Genomics 2015, 16 (Suppl. 5), S3. [CrossRef]

16. Hao, D.; Li, J.; Jia, S.; Meng, Y.; Zhang, C.; Wang, L.; Di, L.J. Integrated Analysis Reveals Tubal- and Ovarian-Originated Serous Ovarian Cancer and Predicts Differential Therapeutic Responses. Clin. Cancer Res. 2017, 23, 7400-7411. [CrossRef]

17. Normandin, K.; Peant, B.; Le Page, C.; de Ladurantaye, M.; Ouellet, V.; Tonin, P.N.; Provencher, D.M.; Mes-Masson, A.M. Protease inhibitor SERPINA1 expression in epithelial ovarian cancer. Clin. Exp. Metastasis 2010, 27, 55-69. [CrossRef] [PubMed]

18. Timms, J.F.; Arslan-Low, E.; Kabir, M.; Worthington, J.; Camuzeaux, S.; Sinclair, J.; Szaub, J.; Afrough, B.; Podust, V.N.; Fourkala, E.O.; et al. Discovery of serum biomarkers of ovarian cancer using complementary proteomic profiling strategies. Proteomics Clin. Appl. 2014, 8, 982-993. [CrossRef]

19. Wu, W.; Wang, Q.; Yin, F.; Yang, Z.; Zhang, W.; Gabra, H.; Li, L. Identification of proteomic and metabolic signatures associated with chemoresistance of human epithelial ovarian cancer. Int. J. Oncol. 2016, 49, 1651-1665. [CrossRef]

20. Yeo, S.K.; French, R.; Spada, F.; Clarkson, R. Opposing roles of Nfkb2 gene products p100 and p52 in the regulation of breast cancer stem cells. Breast Cancer Res. Treat. 2017, 162, 465-477. [CrossRef]

21. Uno, M.; Saitoh, Y.; Mochida, K.; Tsuruyama, E.; Kiyono, T.; Imoto, I.; Inazawa, J.; Yuasa, Y.; Kubota, T.; Yamaoka, S. NF-kappaB inducing kinase, a central signaling component of the non-canonical pathway of NF-kappaB, contributes to ovarian cancer progression. PLoS ONE 2014, 9, e88347. [CrossRef]

22. Shen, K.; Rice, S.D.; Gingrich, D.A.; Wang, D.; Mi, Z.; Tian, C.; Ding, Z.; Brower, S.L.; Ervin, P.R., Jr.; Gabrin, M.J.; et al. Distinct genes related to drug response identified in ER positive and ER negative breast cancer cell lines. PLoS ONE 2012, 7, e40900. [CrossRef] [PubMed]

23. Irjala, H.; Salmi, M.; Alanen, K.; Grenman, R.; Jalkanen, S. Vascular adhesion protein 1 mediates binding of immunotherapeutic effector cells to tumor endothelium. J. Immunol. 2001, 166, 6937-6943. [CrossRef] [PubMed] 
24. Lai, Y.C.; Chang, S.J.; Kostoro, J.; Kwan, A.L.; Chai, C.Y. Vascular adhesion protein-1 as indicator of breast cancer tumor aggressiveness and invasiveness. APMIS 2018, 126, 755-761. [CrossRef] [PubMed]

25. Gharpure, K.M.; Pradeep, S.; Sans, M.; Rupaimoole, R.; Ivan, C.; Wu, S.Y.; Bayraktar, E.; Nagaraja, A.S.; Mangala, L.S.; Zhang, X.; et al. FABP4 as a key determinant of metastatic potential of ovarian cancer. Nat. Commun. 2018, 9, 2923. [CrossRef]

26. Poruk, K.E.; Firpo, M.A.; Huerter, L.M.; Scaife, C.L.; Emerson, L.L.; Boucher, K.M.; Jones, K.A.; Mulvihill, S.J. Serum platelet factor 4 is an independent predictor of survival and venous thromboembolism in patients with pancreatic adenocarcinoma. Cancer Epidemiol. Biomark. Prev. 2010, 19, 2605-2610. [CrossRef]

27. Siamakpour-Reihani, S.; Owzar, K.; Jiang, C.; Turner, T.; Deng, Y.; Bean, S.M.; Horton, J.K.; Berchuck, A.; Marks, J.R.; Dewhirst, M.W.; et al. Prognostic significance of differential expression of angiogenic genes in women with high-grade serous ovarian carcinoma. Gynecol. Oncol. 2015, 139, 23-29. [CrossRef]

28. Piver, M.S.; Moyer, M.; Diakun, K.; Lele, S.B.; Chu, T.M. Serum alpha 1-acid glycoprotein in epithelial ovarian cancer. Gynecol. Oncol. 1988, 29, 305-308. [CrossRef]

29. Moore, L.E.; Pfeiffer, R.M.; Zhang, Z.; Lu, K.H.; Fung, E.T.; Bast, R.C., Jr. Proteomic biomarkers in combination with CA 125 for detection of epithelial ovarian cancer using prediagnostic serum samples from the Prostate, Lung, Colorectal, and Ovarian (PLCO) Cancer Screening Trial. Cancer 2012, 118, 91-100. [CrossRef]

30. Collinson, F.; Hutchinson, M.; Craven, R.A.; Cairns, D.A.; Zougman, A.; Wind, T.C.; Gahir, N.; Messenger, M.P.; Jackson, S.; Thompson, D.; et al. Predicting response to bevacizumab in ovarian cancer: A panel of potential biomarkers informing treatment selection. Clin. Cancer Res. 2013, 19, 5227-5239. [CrossRef]

31. Moore, R.G.; Blackman, A.; Miller, M.C.; Robison, K.; DiSilvestro, P.A.; Eklund, E.E.; Strongin, R.; Messerlian, G. Multiple biomarker algorithms to predict epithelial ovarian cancer in women with a pelvic mass: Can additional makers improve performance? Gynecol. Oncol. 2019, 154, 150-155. [CrossRef] [PubMed]

32. Lee, H.; Kim, K.; Woo, J.; Park, J.; Kim, H.; Lee, K.E.; Kim, Y.; Moon, K.C.; Kim, J.Y.; Park, I.A.; et al. Quantitative Proteomic Analysis Identifies AHNAK (Neuroblast Differentiation-associated Protein AHNAK) as a Novel Candidate Biomarker for Bladder Urothelial Carcinoma Diagnosis by Liquid-based Cytology. Mol. Cell. Proteomics 2018, 17, 1788-1802. [CrossRef] [PubMed]

33. Wisniewski, J.R.; Gaugaz, F.Z. Fast and sensitive total protein and Peptide assays for proteomic analysis. Anal. Chem. 2015, 87, 4110-4116. [CrossRef] [PubMed]

34. Kulak, N.A.; Pichler, G.; Paron, I.; Nagaraj, N.; Mann, M. Minimal, encapsulated proteomic-sample processing applied to copy-number estimation in eukaryotic cells. Nat. Methods 2014, 11, 319-324. [CrossRef] [PubMed]

35. Tyanova, S.; Temu, T.; Cox, J. The MaxQuant computational platform for mass spectrometry-based shotgun proteomics. Nat. Protoc. 2016, 11, 2301-2319. [CrossRef] [PubMed]

36. Cox, J.; Neuhauser, N.; Michalski, A.; Scheltema, R.A.; Olsen, J.V.; Mann, M. Andromeda: A peptide search engine integrated into the MaxQuant environment. J. Proteome Res. 2011, 10, 1794-1805. [CrossRef]

37. Schwanhausser, B.; Busse, D.; Li, N.; Dittmar, G.; Schuchhardt, J.; Wolf, J.; Chen, W.; Selbach, M. Global quantification of mammalian gene expression control. Nature 2011, 473, 337-342. [CrossRef]

38. Tyanova, S.; Temu, T.; Sinitcyn, P.; Carlson, A.; Hein, M.Y.; Geiger, T.; Mann, M.; Cox, J. The Perseus computational platform for comprehensive analysis of (prote) omics data. Nat. Methods 2016, 13, 731-740. [CrossRef]

39. Deeb, S.J.; Tyanova, S.; Hummel, M.; Schmidt-Supprian, M.; Cox, J.; Mann, M. Machine Learning-based Classification of Diffuse Large B-cell Lymphoma Patients by Their Protein Expression Profiles. Mol. Cell. Proteomics 2015, 14, 2947-2960. [CrossRef]

40. Supek, F.; Bosnjak, M.; Skunca, N.; Smuc, T. REVIGO summarizes and visualizes long lists of gene ontology terms. PLoS ONE 2011, 6, e21800. [CrossRef]

41. Yoshihara, K.; Shahmoradgoli, M.; Martínez, E.; Vegesna, R.; Kim, H.; Torres-Garcia, W.; Treviño, V.; Shen, H.; Laird, P.W.; Levine, D.A.; et al. Inferring tumour purity and stromal and immune cell admixture from expression data. Nat. Commun. 2013, 4, 2612. [CrossRef] [PubMed]

(C) 2020 by the authors. Licensee MDPI, Basel, Switzerland. This article is an open access article distributed under the terms and conditions of the Creative Commons Attribution (CC BY) license (http://creativecommons.org/licenses/by/4.0/). 